Thomas Ahrend

\title{
Das Verhältnis von Musik und Sprache bei Nietzsche
}

In einem Fragment aus dem Winter 1869/70 schreibt Nietzsche: "Die Musik ist eine Sprache, die einer unendlichen Verdeutlichung fähig ist." (N 1869/70, 2 [10]) $)^{1}$ Knapp zwanzig Jahre später, in Der Fall Wagner, wird dieser Gedanke zu einer Kritik gegen Wagner gewendet:

"Wagner war nicht Musiker von Instinkt. Dies bewies er damit, dass er alle Gesetzlichkeit und, bestimmter geredet, allen Stil in der Musik preisgab, um aus ihr zu machen, was er nöthig hatte, eine Theater-Rhetorik [...]. Wagner dürfte uns hier als Erfinder und Neuerer ersten Ranges gelten - er hat das Sprachvermögen der Musik in's Unermessliche vermehrt - : er ist der Victor Hugo der Musik als Sprache. Immer vorausgesetzt, dass man zuerst gelten lässt, Musik dürfe unter Umständen nicht Musik, sondern Sprache, sondern Werkzeug, sondern ancilla dramaturgica sein." (WA, KSA 6, 30)

Wie verläuft dieser Wandel in Nietzsches Auffassung über das Verhältnis von Musik und Sprache und die damit verbundene Kritik an Wagner im einzelnen? Kurz: Was dachte Nietzsche über die Beziehung von Musik und Sprache?

Zwei Vorbemerkungen:

1) Das Verhältnis von Musik und Sprache möchte ich nicht als musikästhetisches Problem - Ästhetik hier (verkürzend) verstanden als eine Tätigkeit, die sich als Erkenntnisziel Werturteile setzt -, sondern als musikphilosophisches Problem fassen; Musikphilosophie - also die begriffliche Klärung musikalischer Phänomene - geht nicht in Musikästhetik auf, auch wenn die Tatsache, daß die Wert-

1 Zitatangaben in Klammern beziehen sich immer auf Friedrich Nietzsche, Sämtliche Werke, Kritische Studienausgabe in 15 Bänden, hg. v. Giorgio Colli u. Mazzino Montinari, München/Berlin/New York 1980 (= KSA). Die Siglen sind die dort im kritischen Apparat verwendeten; Fragmente aus dem Nachlaß bezeichne ich mit der Sigle N, Jahreszahl und Fragmentnummer. 
urteile den größten Teil dessen darstellen, was in öffentlichen Diskursen an Musikphilosophie betrieben wird, das Gegenteil suggeriert. Trotzdem lassen sich natürlich musikphilosophische Themen für die Musikästhetik fruchtbar machen, indem sie normativ gewendet werden (z.B.: "Musik soll eine Sprache sein" vs. "Musik soll keine Sprache sein"). Dies geschieht auch bei Nietzsche, wie das Zitat aus Der Fall Wagner zeigt; in einer Untersuchung über das Verhältnis von Musik und Sprache bei Nietzsche wird man immer wieder mit dessen Auseinandersetzung mit Wagner konfrontiert. Mir geht es in diesem Fall nicht um die verschiedenen Aspekte der Begründung von Nietzsches Werturteilen, sondern um die systematischen Positionen zum Verhältnis zwischen Musik und Sprache, die sich am Beispiel seiner Wagnerkritik herausbilden lassen.

2) Das musikphilosophische Problem des Verhältnisses zwischen Musik und Sprache (enger gefaßt: Inwiefern ist Musik eine Sprache? und: Inwiefern ist Sprache Musik?) ist nicht zu verwechseln - wenn auch eng verbunden - mit

a) den Problemen, die sich aus dem konkreten Zusammenwirken von Musik und Sprache ergeben (in vertonten Texten oder in mit Text unterlegter Musik), und

b) mit der Problematik "absolute Musik" vs. "Programmusik" bzw. "dramatische Musik".

Auch hier gilt, daß ich an Texten, welche diese Probleme - obwohl sie nicht direkter Gegenstand meiner Untersuchung sind - an der Oberfläche verhandeln, Positionen herauszuarbeiten versuchen werde, die für meine eigentliche Problemstellung relevant sind.

Ich sehe in Nietzsches Haltung zum Verhältnis zwischen Musik und Sprache drei Phasen: Die erste Phase $^{2}$ (I) umfaßt den Zeitraum der Entstehung von Die Geburt der Tragödie und Richard Wagner in Bayreuth; die zweite Phase (II) ist ein radikaler Bruch mit den Positionen der ersten Phase, der sich vor allem in Menschliches, Allzumenschliches dokumentiert; die dritte Phase schließlich (III) umfaßt die gesamten achtziger Jahre.

2 Ich beginne meine Untersuchung mit der Abfassung der Geburt der Tragödie, lasse also die Jugendschriften Nietzsches außer Betracht, da diese, so wichtig sie zum Verständnis der Voraussetzungen und Motivationen der Philosophie Nietzsches generell sind, in - auf meine Fragestellung bezogen - begrifflich-systematischer Hinsicht keine Positionen hinzufügen, die nicht auch später und dann deutlicher formuliert worden wären. 


\section{Die Geburt der Tragödie und Richard Wagner in Bayreuth}

In der Zeit der Entstehung von und in Die Geburt der Tragödie ist Nietzsche, beeinflußt durch Wagner und Schopenhauer, offensichtlich einer romantischen Anschauung verpflichtet: dem Unsagbarkeits-Topos. Musik ist eine Sprache, die ausspricht, was eigentlich nicht auszusprechen ist. Für die deutsche Frühromantik und noch E.T.A. Hoffmann war dies "das Absolute" bzw. "Geist"; für Nietzsche ist es der "Wille", das "Dionysische" oder das "Ur-Eine": Die Musik ist "recht eigentlich die Sprache des Allgemeinen" (N 1871, 9 [88]), sie hat einen "dionysischen Inhalt", der in der Musik als "Wille" erscheint (Vgl. GT 6, KSA $1,50)$ und "sich auf den Urwiderspruch und Urschmerz im Herzen des Ur-Einen symbolisch bezieht" (ebd., 51). Die Musik ist der Ursprung aller Sprache, ist immer allgemeiner als jede Wortsprache, und treffen Musik und Sprache zusammen, wie im Lied oder in der Oper, so ist der Text immer nur ein Beispiel für das Allgemeine, das die Musik ist.

Diese Haltung steht im großen und ganzen im Einklang mit der metaphysisch ausgezeichneten Rolle der Musik bei Schopenhauer und Wagner (insofern dieser sich auf Schopenhauer bezieht); im Detail zeigen sich aber hier schon gewisse Dissonanzen: 1871 befürwortet Nietzsche in einem Fragment (N 1871, 12 [1]) Über Musik und Wort die "absolute Musik" in einem Maße, die mit Wagners "älterer Lehre" (Musik als Mittel des Dramas) nicht übereinstimmt. Die "neuere Lehre" (Wagners Beethoven-Schrift: Drama als Beispiel für das Allgemeine der Musik) fordert aber laut Nietzsche als Konsequenz: "auch die Musik des Drama's muss absolute Musik sein" (N 1874, 32 [52], KSA 7, 770), was Nietzsche spätestens seit 1874 in Wagners Musik - also nicht bloß in dessen theoretischen Schriften vor der Schopenhauer-Lektüre - nicht mehr gewährleistet sieht:

"Jetzt ist wirklich die Musik ein 'Mittel des Ausdrucks' geworden: steht deshalb künstlerisch auf einer niedern Stufe, denn sie ist nicht mehr organisch in sich." (Ebd., 773)

Diese von Carl Dahlhaus an verschiedenen Stellen beschriebene Problematik ${ }^{4}$ zwischen "absoluter" und "dramatischer" Musik und die daraus resultierende

3 Dieser Titel stammt von späteren Herausgebern und fehlt deswegen zurecht in KGW und KSA; da er aber den Inhalt des Fragments wiedergibt und sich in der musikwissenschaftlichen Literatur eingebürgert hat, verwende ich ihn zur Bezeichnung des Fragmentes.

4 Vgl. Carl Dahlhaus, "Die doppelte Wahrheit in Wagners Ästhetik. Zu Nietzsches Frag- 
Differenz zwischen Nietzsche und Wagner ist eigentlich schon die Fortsetzung einer Problematik, die in Nietzsches bisheriger Auffassung von dem Verhältnis zwischen Musik und Sprache liegt. Sprache ist gegenüber der Musik schon defizient, ein Verfallsprodukt, weil die Symbolik, welche die Musik generiert, in der Worte und Begriffe verwendenden Sprache nur noch "gebraucht" wird: "Ein gemerktes Symbol ist immer ein Begriff: man begreift, was man bezeichnen und unterscheiden kann" (N 1870, 3 [15]); Begriffe sind demnach die "apollinischen Spiegelungen des dionysischen Grundes" (N 1870, 8 [41]); und: "In sofern ist der Begriff der Tod der Kunst, als er sie zum Symbol herabzieht." (N 1871, 9 [88])

Ist es dann aber überhaupt wünschenswert, daß das Unsagbare, das Dionysische mittels der Musik gesagt wird, da dadurch Musik selbst zu Sprache - und somit Verfallsprodukt - wird? "Ein Glück, daß die Musik nicht spricht - obschon jetzt die Musiker viel schwätzen." (N 1872, 19 [264]) Das metaphysisch bedingte Ideal von Musik spricht nicht, die musikalische Realität - zumal die Wagners - spricht sehr wohl.

In Richard Wagner in Bayreuth wird dieser Widerstreit schon deutlicher, wenn auch nicht gänzlich explizit: Was im veröffentlichten Text in bezug auf Wagners Musik als "tönende[] Processe des Gefühls und der Leidenschaft" (WB 9, KSA 1, 493) verklausuliert ist, liest sich in der Vorstufe folgendermaßen: "Er [Wagner; T.A.] hat erreicht, was noch nie einer erreicht hat: die allerstärkste und deutlichste Sprache des Gefühls." (N 1875, 11 [15], KSA 8, 199) Das wurde aber eigentlich schon in dem erwähnten Fragment Über Musik und Wort von 1871 als nicht charakteristisch für die Musik angesehen:

"[...] das Gefühl [...] ist überhaupt im Bereich der produktiven Kunst das an sich Unkünstlerische, ja erst seine gänzliche Ausschließung ermöglicht das volle sich Versenken und interesselose Anschauen des Künstlers. [...] Das, was wir Gefühle nennen, ist [...] nicht mehr direkt Gegenstand der Musik: geschweige denn, daß es diese aus sich erzeugen könnte." (N 1871, 12 [1], KSA 7, 364)

Wenn in Richard Wagner in Bayreuth die Musik Wagners als eine Sprache des Gefühls gepriesen wird, so ist dies vielleicht Nietzsches Art, Wagner auf die

ment 'Über Musik und Wort'", in ders., Zwischen Romantik und Moderne. Vier Studien zur Musikgeschichte des späteren 19. Jahrhunderts, Berliner Musikwissenschaftliche Arbeiten, Bd. 7, München 1974, 22-37; ders., Die Idee der absoluten Musik, Kassel/München 1978, 35-40. 
Problematik aufmerksam zu machen, ${ }^{5}$ zumal Nietzsche die bedenklichen Konsequenzen, die sich seiner Meinung nach daraus für die Musik ergeben, durchaus nennt:

"[...] genug, wenn wir es an einzelnen Fällen spüren, wie seine [Wagners; T.A.] Musik sich mit einer gewissen Grausamkeit des Entschlusses dem Gange des Dramas, der wie das Schicksal unerbittlich ist, unterwirft, während die feurige Seele dieser Kunst darnach lechzt, einmal ohne alle Zügel in der Freiheit und Wildnis umherzuschweifen." (WB 9, KSA 1, 496)

\section{Menschliches, Allzumenschliches}

In Menschliches, Allzumenschliches bricht Nietzsche dann explizit mit der Auffassung Schopenhauers, Wagners und seiner eigenen älteren: Musik ist keine unmittelbare Sprache des Gefühls, sie spricht nicht vom "Willen" oder "Dinge an sich", und wenn ihr eine "Bedeutsamkeit" zugesprochen wird, so ist dies nur möglich mittels einer "Symbolik" (vgl. MA 215), die sich genau so der "Convention" verdankt (vgl. WS 122), wie das bei der Wortsprache der Fall ist:

"Die Musik ist eben nicht eine allgemeine überzeitliche Sprache, wie man so oft zu ihrer Ehre gesagt hat, sondern entspricht genau einem Gefühls-, Wärme- und Zeitmaass, welches eine ganz bestimmte einzelne, zeitlich und örtlich gebundene Cultur als inneres Gesetz in sich trägt [...]" (VMS 171, KSA 2, 450)

Daraus ergeben sich zwei Konsequenzen:

1) Verschiedene Menschen mit unterschiedlichem musikalischen "Background" sowie einzelne Individuen zu unterschiedlichen Zeitpunkten und Stimmungslagen (vgl. WS 168 und N 1881, 12 [16]) hören und interpretieren ein und dasselbe Stück Musik unterschiedlich:

"[D]ie Musik Palestrina's würde für einen Griechen völlig unzugänglich sein, und wiederum - was würde Palestrina bei der Musik Rossini's hören? Vielleicht, dass auch unsere neueste deutsche Musik [...] in kurzer Zeitspanne nicht mehr verstanden wird [...]" (VMS 171, KSA 2, 450)

5 Diese Lesart von WB deckt sich mit der von Montinari vorgeschlagenen. Vgl. Mazzino Montinari, "Nietzsche und Wagner vor hundert Jahren", Nietzsche Studien, 7 (1978), 288-302. 
2) Der Unsagbarkeits-Topos erfährt eine Relativierung, die praktisch zu dessen Umkehrung führt: Musik spricht nicht das schlechthin Unsagbare aus, sondern das bisher Ungesagte:

"Unsäglich 'vieles' hat noch kein Wort gefunden und keinen Gedanken - das beweist unsere Musik - nicht, daß kein Gedanke und kein Wort da zu finden wäre." (N 1881, 12 [36])

Das führt bei Nietzsche zu einer Aufwertung der Wortsprache und des begrifflichen Denkens, die sich konträr zu seiner Hochschätzung der Musik in Die Geburt der Tragödie verhält und eher dem Voltaireschen, literarischen Geist von Menschliches, Allzumenschliches entspricht. In einem Fragment aus dem Jahre 1880 wird dies sehr deutlich formuliert:

"Die Musik hat keinen Klang für die Entzückungen des Geistes [...]. Sie [die Musiker; T.A.] müssen alle Zustände vergröbern, gleichsam ins Unmenschliche zurückübersetzen: wie als ob die Gedanken und die Worte noch nicht erfunden seien. [...] Der Dichter steht höher als der Musiker, er macht höhere Ansprüche, nämlich an den ganzen Menschen: und der Denker macht noch höhere Ansprüche: er will die ganze ges < ammelte > frische Kraft und fordert nicht zum Genießen sondern zum Ringkampf und zur tiefsten Entsagung aller persönlichen Triebe auf." (N 1880, 6 [39])

Im Vergleich dazu - noch 1878 heißt es:

"Beethoven hat es besser gemacht als Schiller. Bach besser als Klopstock. Mozart besser als Wieland. Wagner besser als Kleist." (N 1878, 27 [93])

Daß - so ist diese Äußerung im einzelnen vermutlich zu verstehen - Beethovens 9. Sinfonie der Schillerschen Ode, welche in ihr vertont ist, überlegen sei, daß die Bachschen Oratorien den Messias von Klopstock an Religiosität, die Mozartschen Musikdramen die Romane und Versepen Wielands an erotischem Witz und Wagners Meistersinger - als "Komödie" - den Zerbrochenen Krug von Kleist ${ }^{6}$

6 Freilich bleiben diese Zuordnungen einzelner Werke spekulativ. Der Vergleich zwischen Wagner und Kleist jedoch ließe noch eine weitere Zuordnung $\mathrm{zu}$, die bei Nietzsche ansatzweise - allerdings erst 1888 - belegbar ist: Das "hysterisch-heroische Weib" als Typus bei Wagner ("Elsa, Senta, Isolde, Brünnhilde, Kundry") wurde laut Nietzsche "in Deutschland nicht gänzlich degoutiert", weil "bereits [...] der edle Heinrich von Kleist 
übertreffen, ist eine romantische Position, deren metaphysische Begründung in der Geburt der Tragödie versucht worden war, die sich aber als nicht haltbar erwies. Nietzsche bezieht in Menschliches, Allzumenschliches nun eine radikale Gegenposition, deren Begründung empirisch ausfällt; die jeweils ersten Aphorismen eines Hauptstückes zeigen dies an: In der selben Hinsicht, wie eine "Chemie der Begriffe und Empfindungen" (MA, 1) und die "psychologische[] Beobachtung П" (MA, 35) die philosophische Betrachtungsweise von Metaphysik und Moral verändern, wenn nicht gar ersetzen sollen, wird von der "Wissenschaft der Kunst" gefordert, der künstlerischen "Illusion [...] auf das bestimmteste zu widersprechen und die Fehlschlüsse und Verwöhnungen des Intellects aufzuzeigen, vermöge welcher er dem Künstler in das Netz läuft" (MA, 145). Die romantische Konzeption der Musik als überhöhte bzw. eigentliche Sprache wird dann von Nietzsche (Vgl. MA 215) als eine solche Illusion bestimmt.

Allerdings bleibt er nicht bei dieser radikalen Gegenposition stehen.

\section{Die achtziger Jahre}

Diese beiden konträren Positionen - auf der einen Seite die metaphysische Hochschätzung der Musik gegenüber der Sprache in Die Geburt der Tragödie; auf der anderen Seite die Aufwertung der Sprache gegenüber der Musik in Menschliches, Allzumenschliches - werden in den achtziger Jahren - bis zum geistigen Zusammenbruch Nietzsches - versucht zu vermitteln, was zu drei Konsequenzen führt:

1) Die Musik ist eine Sprache unter vielen: "Es gibt vielmehr Sprachen als man denkt: [...] Was redet nicht!" (N 1883, 7 [62], KSA 10, 262) Sie besitzt zwar Besonderheiten, die es z.B. immer noch erlauben das Dionysische in ihre Nähe zu rücken, sie allerdings nicht mehr metaphysisch auszeichnen. Sie ist "eine Zeichensprache der Affekte", und der Musiker verrät in ihr sein "Triebsystem", sofern er "unschuldig" (ebd.) ist. (Nietzsche entdeckt auch bei Wagner immer noch solche "unschuldigen" Momente: "Ich bewundere Wagner in Allem, worin er sich in Musik setzt. -" [NW, KSA 6, 418]) Die "Affekte" selbst sind aber "eine Construction des Intellekts" (N 1883, 24 [20]), also intellektuell vermittelt, und Musik ist "ein Nachbild vom Nachbild von Gefühlen" (M, 142; KSA 3, 133). Eine Musik, die sich "schuldig" macht, diesen Zeichenprozeß zu unterlaufen, indem sie von vornherein darauf hin konzipiert wird, wie der Zuhörer sie deuten soll, also "schauspielert", wird von Nietzsche kritisiert:

[...] ihm [Wagner] daselbst die Fürsprache des Genies gegeben hatte" (N 1888, 14 [63];

N 1888, 16 [48]). 
"Wagner hat keine Gedanken, ganz wie V. Hugo: aber er weiß mit einem Zeichen an Stelle eines Gedankens uns dermaaßen zu terrorisiren - - -" (N 1888,15 [12])

Der Gegensatz zwischen "unschuldiger und schuldiger Musik" (M 255, KSA 3, 207) oder "monologischer Kunst" und "Kunst vor Zeugen" (vgl. FW, 367) gliedert also das Verhältnis von Musik und Sprache begrifflich in zwei mögliche Fälle:

a) die Musik kann einen, wenn auch komplex strukturierten, aber unmittelbaren Zeichenprozeß generieren oder

b) dieser Prozeß ist fingiert und wird so Mittel zum Zweck, schränkt damit die Interpretationsmöglichkeiten des Rezipienten ein, tyrannisiert ihn.

Diese Unterscheidung wird allerdings in einem "Gespräch über Musik" (M 255, KSA 3, 206-208) betitelten Text der Morgenröthe sofort wieder relativiert:

2) Alle Musik ist wieder interpretierbar, auch hinsichtlich ihres Sprachcharakters. In dem Dialog "Gespräch über Musik" beschreibt ein Zuhörer A einem Zuhörer B ein Stück Musik, während dieses erklingt, als Schauspieler-Musik. Diese Beschreibung bringt B aus der Fassung und läßt ihn beinahe verzweifeln:

"[I]ch kann es nicht mehr ertragen, Sie zu hören! Zehnmal lieber will ich doch mich täuschen lassen, als Einmal in ihrer Art die Wahrheit zu wissen!" (Ebd., 207)

Dann erklärt A jedoch, nachdem er seine Vorstellung einer "unschuldigen" Musik beschrieben hat, seine ganze Beschreibung sei erlogen gewesen:

"[I]ch nenne eine unschuldige Musik jene, welche ganz und gar nur an sich denkt, an sich glaubt, und über sich die Welt vergessen hat, - das Von-selberErtönen der tiefsten Einsamkeit, die über sich mit sich redet und nicht mehr weiss, dass es Hörer und Lauscher und Wirkungen und Missverständnisse und Misserfolge da draussen giebt. - Zuletzt: die Musik, welche wir eben hörten, ist gerade von dieser edlen und seltenen Art, und Alles, was ich von ihr sagte, war erlogen, - verzeihen Sie meine Bosheit, wenn Sie Lust haben!" (Ebd., 207 f.)

Das heißt, ob die Zeichensprache einer Musik unmittelbar oder fingiert ist, bleibt genauso den interpretatorischen Möglichkeiten oder Beschränkungen des Zuhörers überlassen wie die mögliche "Bedeutsamkeit" der Musik. 
Hier muß sich Nietzsche fragen lassen, inwiefern er seine Interpretation von Wagners Musik gegenüber anderen möglichen ausgezeichnet wissen will. ${ }^{7}$ Welche Maßstäbe wendet er an, um diese einzelnen, kurzen Takte der Musik Wagners, in denen er "von sich spricht", von der Schauspieler-Musik zu unterscheiden? Immanent musikalische Kriterien ${ }^{8}$ sind es nicht:

"Wir entbehren in der Musik einer Ästhetik, die den Musikern Gesetze aufzuerlegen verstünde und ein Gewissen schüfe; wir entbehren, was eine Folge davon ist, eines eigentlichen Kampfes um 'Principien' - denn als Musiker lachen wir über die Herbartschen Velleitäten auf diesem Gebiete ebenso sehr, als über die Schopenhauers. [...] [W]ir wissen die Begriffe 'Muster', 'Meisterschaft', 'Vollkommenheit' nicht mehr zu begründen - wir tasten mit dem Instinkte alter Liebe und Bewunderung blind herum im Reich der Werthe, wir glauben beinahe 'gut ist was uns gefällt'..." (N 1888, 16 [29], KSA 13, 489 f.)

Vielleicht, daß durch das Fehlen von Kriterien überhaupt - derer es ja bedürfte, um zu Werten zu gelangen - das Verhältnis von Musik und Sprache zwischen den beiden Positionen ambivalent hin und her schwankt:

Auf der einen Seite wird die begriffslose, sinnliche Musik als Narkotikum entlarvt:

"Daß die Musik vom Worte vom Begriffe absehen darf - oh wie sie daraus ihren Vortheil zieht, diese arglistige Heilige, die zu allem zurückführt, $z u$ rückverführt, was einst geglaubt wurde!" (N 1888, 14 [42])

Auf der anderen Seite wird die Musik - auch und immer noch die Wagners gegenüber der Sprache bewundert:

7 Das Problem scheint mir zu korrespondieren mit dem, was Volker Gerhardt - auf einer allgemeineren Ebene - die "Perspektive des Perspektivismus" genannt hat. Vgl. Volker Gerhardt, "Perspektive des Perspektivismus", Nietzsche-Studien, 18 (1989), 260-281.

8 Auch der Briefwechsel mit Carl Fuchs scheint die in ihn gesetzten Hoffnungen ("Da erweckt nun das aufgezeichnete Schema, welches ihr Brief enthält, alle meine Hoffnungen: gerade so einfach thatsächlich müßte es [Wagners Musik] beschrieben werden" [Nietzsche an Carl Fuchs, 29. Juli 1877, zit. nach Friedrich Nietzsche, Sämtliche Briefe, Kritische Studienausgabe in 8 Bänden, hg. v. Giorgio Colli u. Mazzino Montinari, München $1986(=\mathrm{KSB})$, Bd. 5, 262]) in dieser Hinsicht nicht erfüllt zu haben; elf Jahre später formuliert Nietzsche gegenüber Fuchs die Möglichkeit eines musikalischen Werturteils nur noch in der Vergangenheit: "Ich hörte von Niemandem lieber Werthurteile de rebus musicis et musicantibus." (14. April 1888; KSB 8, 295.) 
"Im Verhältniß zur Musik ist alle Mittheilung durch Worthe von schamloser Art; das Wort verdünnt und verdummt; das Wort entpersönlicht: das Wort macht das Ungemeine gemein." (N 1886, 10 [60])

3) Die dritte Konsequenz läßt sich wiederum als Vermittlungsversuch verstehen; Nietzsche möchte Sprache und Philosophie zu Musik werden lassen:

"[...] hinter allen den kalten Worten bewegt sich eine begehrende Seele, ich höre sie singen, denn meine eigene Seele singt, wenn sie bewegt ist." ( $\mathrm{N}$ $1880,7[18])$

Eine Vorstellung, die zu Äußerungen Nietzsches führt, daß Also sprach Zarathustra unter die Musik zu rechnen sei (Vgl. EH, KSA 6, 335), daß, was in der Geburt der Tragödie geschrieben wurde, besser hätte gesungen werden sollen (Vgl. GT, Versuch einer Selbstkritik, KSA 1, 15) usw.

Hiermit kehrt Nietzsche wieder zu dem romantischen Unsagbarkeits-Topos zurück, den er in Menschliches, Allzumenschliches eigentlich in sein Gegenteil verkehrt hatte: Historisch geht nämlich - wie Carl Dahlhaus gezeigt hat - die Vorstellung, daß Musik etwas ausdrückt, was sich in der Sprache nicht sagen läßt, nicht auf die Musik oder Musiker selbst zurück, sondern auf die Dichter:

"Die Entdeckung, daß die Musik, und zwar als gegenstands- und begriffslose Instrumentalmusik, eine Sprache 'über' der Sprache sei, ereignete sich, paradox genug, 'in' der Sprache: in der Dichtung."

$\mathrm{Zu}$ fragen wäre, inwiefern Nietzsche in diesem Punkt selbst immer "Romantiker" gewesen und geblieben ist.

Hier müßte eine Analyse vor allem der Lyrik Nietzsches ansetzen, z.B. des Nachtliedes aus dem Zarathustra, das Nietzsche selbst in Ecce homo als Beispiel für seine Neuerung in der Behandlung der Sprache rühmt (Vgl. EH, Also sprach Zarathustra 7), oder der Dionysos-Dythyramben. Ich möchte kurz auf das Gedicht "An der Brücke stand" (EH, KSA 6, 291; NW, KSA 6, 421) - jene "Seite 'Musik' über Musik"10 - eingehen, um die angesprochene Romantiker-Problematik zu veranschaulichen:

9 Carl Dahlhaus, Die Idee der absoluten Musik, 66.

10 Nietzsche an Carl Fuchs, 27. Dezember 1888; KSB 8, 554. 
An der Brücke stand

Jüngst ich in brauner Nacht.

Fernher kam Gesang:

goldener Tropfen quoll's

über die zitternde Fläche weg.

Gondeln, Lichter, Musik -

trunken schwamm's in die Dämmrung hinaus...

Meine Seele, ein Saitenspiel, sang sich, unsichtbar berührt, heimlich ein Gondellied dazu, zitternd vor bunter Seligkeit.

- Hörte Jemand ihr zu?...

Ich habe nicht vor, eine auch nur annähernd vollständige Interpretation dieses Gedichtes zu liefern; ${ }^{11}$ mir geht es hier nur um die Aspekte, die es ermöglichen, die Problematik zu verdeutlichen.

Das Gedicht weist eine Irritation auf: Der "Gesang" motiviert die "Seele" des lyrischen Ichs auf eine unmerkliche Weise ("unsichtbar berührt"), ein "Gondellied" zu singen. Da aber anzunehmen ist, daß der "Gesang" selbst schon ein "Gondellied" ist ("Gondeln, Lichter, Musik -"), ist es irritierend, daß das "Gondellied" der "Seele" "dazu" gesungen wird und nicht in einem bloßen Mitsingen des gehörten "Gesang[s]" besteht. Das "Gondellied" der Seele scheint auf einer anderen ("heimlich[eren]") Ebene als der "Gesang" zu liegen. Das legt die Deutung nahe, daß das "Gondellied", welches das "Saitenspiel" der "Seele" bewegt, das eigene Gedicht ist.

Die Frage, "- Hörte Jemand ihr zu?...", ließe sich in einer romantischen Lesart als der ironische Hinweis auf den Selbstbezug des Gedichtes auffassen, der eine Vereinigung von Produktion und Rezeption voraussetzt: Das "Gondellied" ist ein Produkt, das sich der Rezeption des "Gesangs" verdankt; diese wären aber von einem potentiellen weiteren Rezipienten ("Jemand") vermutlich nicht voneinander zu unterscheiden, da in der aktuellen Situation, die das Gedicht beschreibt, nur der "Gesang" zu hören ist und in der Situation der Rezeption des Gedichtes das "Gondellied" nur als "Gesang" vorstellbar ist. Soll es trotzdem gelingen - liest man also die Schlußzeile als Suggestivfrage -, so müß-

11 Eine ausführlichere Interpretation des Gedichtes und seiner Beziehung zum vorangehenden Prosatext liefert z.B. Wolfgang Groddeck, "'Ein anderes Wort für Musik'. Zu Friedrich Nietzsches Venedig-Gedicht", in: Gedichte und Interpretationen (5). Vom Naturalismus bis zur Jahrhundertmitte, hg. v. Harald Hartung, Stuttgart 1983, 20-32. 
ten "ich" und "Jemand" zu einer Einheit verschmelzen, mithin die romantische Utopie einer Vereinigung von Subjekt und Objekt vollzogen werden.

Im philosophischen Kontext der Zeit, in der Nietzsche das Gedicht schrieb, könnte man die Schlußzeile auch anders lesen: Als tatsächliche Anrede an den potentiellen Leser und Aufforderung, den gelesenen Text zu "interpretieren". ${ }^{12}$ Die beschriebenen Ambivalenzen zwischen "Gesang" und "Gondellied" bzw. "ich" und "Jemand" wären dann ein Hinweis darauf, daß das Gedicht selbst schon eine Interpretation der Situation - und eine Parodie der Mittel, die romantische Lyrik zur Beschreibung einer solchen Situation bietet - ist, die im Gedicht beschrieben wird. Nietzsches Kunstgriff bestünde in dieser Lesart ${ }^{13}$ darin, romantische Topoi ("Meine Seele, ein Saitenspiel"; "Seligkeit") und Verfahrensweisen (subjektive Erlebnisschilderung; Ironie) negativ auf sich selbst anzuwenden.

\section{Ausblick}

Soweit mein kurzer und verkürzender Überblick über das Verhältnis von Musik und Sprache bei Nietzsche.

Nietzsches Positionen zum Verhältnis Musik - Sprache bieten keine abschlieBende Problemlösung; vielmehr scheint er immer wieder bemüht, den Problemen neue Aspekte abzugewinnen. Hierin liegt aber auch die Anregung begründet, diese Probleme weiterzudenken.

Einen weiterführenden Aspekt bietet z.B. der Vergleich mit der Musikphilosophie Theodor W. Adornos. Dessen Wagner-Kritik; die sich in ihren Grundzügen deutlich von Nietzsche herleitet, ${ }^{14}$ unterscheidet sich allerdings in der Auffassung des Verhältnisses Musik - Sprache:

"Wagners musikalisches Bewußtsein unterliegt einer eigentümlichen Rückbildung; es ist, als hätte die Scheu vor der Mimik, die mit der Geschichte der abendländischen Rationalisierung stets stärker wurde und zur Kristallisation

12 Zur Bedeutung der "Interpretation" beim späten Nietzsche vgl. Johann Figl, Interpretation als philosophisches Prinzip, Berlin/New York 1982 und Günter Abel, Nietzsche: Die Dynamik der Willen zur Macht und die ewige Wiederkehr, Berlin/New York 1984.

13 Die Einbeziehung des vorausgehenden Prosatextes in die Interpretation des Gedichtes macht diese Lesart sehr plausibel. Vgl. Wolfgang Groddeck, 22-25, 30.

14 Ohne daß Adorno dies explizit macht, was ihm Nietzsche-Exegeten zuweilen übelnehmen. Vgl. z.B. Franz-Peter Hudek, "Nietzsche im Lichte der Musik-Fachwelt", Nietzsche-Studien, 19 (1990), 230-245, hier 237. 
einer autonomen, sprachähnlichen Logik der Musik nicht wenig beitrug, über ihn nicht die volle Macht. Sein Komponieren fällt zurück auf ein Vorsprachliches, ohne doch dabei des Sprachähnlichen ganz sich entäußern zu können. Die Wagnersche 'Schauspielerei', das Anstößige seines Verfahrens, wie Paul Bekker sie mit Recht als den Kern des Wagnerschen Kunstwerks schlechthin aufgefaßt hat, gründet in jener Regression. ${ }^{15}$

So differenziert Adornos Urteil auch zu lesen ist, in der Tendenz fordert er eine Sprachähnlichkeit, die Nietzsche bei Wagner gerade kritisiert. Adorno hat diese Divergenz erkannt - und vielleicht deswegen im eben zitierten Abschnitt aus dem Versuch über Wagner statt Nietzsche lieber Paul Bekker als Referenz für den Vorwurf der "Schauspielerei" genannt - und an anderer Stelle - nachdem er den Aphorismus 215 aus Menschliches, Allzumenschliches zitiert, in dem Nietzsche davon spricht, daß "[a]n sich [...] keine Musik tief und bedeutungsvoll" ist folgendermaßen formuliert:

"Das von Nietzsche postulierte 'An sich' ist fiktiv: alle neuere Musik konstituiert sich als Bedeutungsträger, hat ihr Sein bloß am Mehr-als-nur-TonSein und läßt darum nicht in Wahn und Wirklichkeit sich zerlegen. Es ist denn auch Nietzsches Begriff des musikalischen Fortschritts als anwachsender Psychologisierung zu geradlinig konzipiert. Weil das Material selber schon Geist ist, bewegt sich die Dialektik der Musik zwischen dem objektiven und subjektiven Pol, und keineswegs kommt diesem, abstrakt, der höhere Rang zu." 16

Auch in musikwissenschaftlichen Texten der letzten Zeit finden sich Ansätze, die sich mit Nietzsches Problemstellungen und Lösungsansätzen berühren: Die aktuellen Bemühungen der amerikanischen Musikwissenschaft um eine "musical hermeneutic" bzw. einen "music criticism" z.B. problematisieren die Möglichkeiten einer musikalischen "Bedeutung" sehr ausführlich.

Susan McClary - exponierte Vertreterin eines feministischen "music criticism" - scheint den Nietzsche von Menschliches, Allzumenschliches zu paraphrasieren, wenn sie schreibt:

15 Theodor W. Adorno, "Versuch über Wagner", in: ders., Die musikalischen Monographien, Frankfurt a.M. 1986, 32.

16 Theodor W. Adorno, Philosophie der neuen Musik, Frankfurt a.M. 1978, 130, Fn. 
"Like any social discourse, music is meaningful precisely insofar as at least some people believe that it is and act in accordance with that belief. Meaning is not inherent in music, but neither is it in language: both are activities that are kept afloat only because communities of people invest in them, agree collectively that their signs serve as valid currency." 17

Damit bezieht McClary indirekt Position gegen Adorno, dessen historiographischen Ansatz - der in seiner Begründung m.E. jedoch nicht von seiner dialektischen Theorie- und Sprachauffassung zu trennen ist - sie allerdings auch für sich in Anspruch nimmt. ${ }^{18}$

Alle diese Positionen einmal im Vergleich begrifflich auf ihren Kern hin zu untersuchen, wäre Stoff für eine größere Arbeit als es die vorliegende sein kann und will.

17 Susan McClary, Feminine Endings: Music, Gender, and Sexuality, Minnesota 1991, 21. $18 \mathrm{Vgl}$. ebd., $28 \mathrm{f}$. 